\title{
Transient reduction in venous susceptibility during Posterior Reversible Encephalopathy Syndrome
}

Morgan Ollivier ${ }^{1,2}$, Emmanuelle Wanono3 ${ }^{3}$ Delphine Leclercq ${ }^{2}$, Fanny Domont ${ }^{3}$, Stéphanie Trunet ${ }^{2}$, Didier Dormont ${ }^{2,4}$, Anne Bertrand ${ }^{2,4}$.

${ }^{1}$ Department of Radiology, University Hospital Center, Hôpital de la Cavale-Blanche, F29609, Brest cedex, France

2 AP-HP, Pitié-Salpétrière Hospital, Department of Functional and Diagnostic Neuroradiology, F-75013, Paris, France

3 AP-HP, Pitié-Salpétrière Hospital, Department of Internal Medicine, F-75013, Paris, France

${ }^{4}$ Sorbonne Universités, UPMC Univ Paris 06 UMR S 1127, Inserm U 1127, CNRS UMR 7225, ICM, Inria Paris-Rocquencourt, F-75013, Paris, France

Corresponding Author:

Anne Bertrand

Service de NeuroradiologieDiagnostiqueetFonctionnelle, HôpitalPitié-Salpêtrière, 47, Boulevard de l'Hôpital, 75651 Paris Cedex 13, France.

Tel: 0033142163596

Fax : 0033142163515

Email: anne.bertrand@aphp.fr 
KEY WORDS: Posterior reversible encephalopathy syndrome (PRES); Magnetic Resonance Imaging (MRI); Blood-oxygen Level Dependent (BOLD); T2*; SWAN; Susceptibility-weighted Imaging (SWI); Arterial spin labeling (ASL).

WORD COUNT: 355

\begin{abstract}
We report a case of Posterior Reversible Encephalopathy Syndrome (PRES) in which followed-up MRI demonstrated a transient reduction in venous signal on initial SWAN images. The progressive normalization of venous signal on D10 and D40 imaging paralleled the progressive decrease of hyperperfusion on CBF images. Decreased venous susceptibility has never been reported in PRES; it relates most likely to a transient BOLD effect induced by brain hyperperfusion.
\end{abstract}




\section{Transient reduction in venous susceptibility during Posterior Reversible Encephalopathy Syndrome}

Posterior reversible encephalopathy syndrome (PRES) is a neurotoxic state promoted by several well-known factors (severe hypertension, pre-eclampsia, cytotoxic and immunosuppressive drugs, autoimmune diseases). During PRES, brain MRI typically demonstrates reversible vasogenic subcortical edema in the posterior regions and anterior watershed regions[1]. Although the mechanism of PRES remains controversial, the main hypothesis postulates that an increase in blood pressure, beyond the upper limit of brain vasculature autoregulation, induces blood vessel alteration, hyperperfusion and vasogenic edema[2].

A 21-year-old woman with systemic lupus erythematous was referred in emergency for acute headaches and hypertensive crisis followed by a tonico-clonic seizure. 3T Brain MRI demonstrated bilateral corticosubcortical FLAIR hyperintensities in the occipitallobes and anterior watershed regions, suggestive of PRES (Figure 1). DWI was normal (not shown). Cerebral Blood Flow map (CBF) generated by Arterial Spin Labelling perfusion (ASL) showed increased CBF in both occipital regions and anterior watershed regions, also suggestive of PRES[2] (Figure 1). On T2 Star Weighted ANgiography (SWAN), the cerebral veinous system was lacking its usual marked hypointensity, and appeared as a subtle hypointensity only. Clinical evolution was quickly favorable under anti-epileptic and anti-hypertensive drugs. Follow-up MRI at 
D10 and D40 showed regression of edema, progressive normalization of the CBF and of the cerebral venous signal on SWAN.

Brain hyperperfusion in the initial stage of PRES is a classical but inconstant finding[2],3. Hyperperfusion is usually associated with an increase in the oxyhemoglobin/deoxyhemoglobin ratio[3]. Because oxyhemoglobin is diamagnetic and deoxyhemoglobin is paramagnetic, this increase in oxyhemoglobin/deoxyhemoglobin ratio leads to an increased signal on $\mathrm{T} 2 *$-weighted sequences, known as the BOLD effect (Blood Oxygenation Level Dependent)[3]. To the best of our knowledge, our case is the first to report a transient BOLD effect during PRES, depictable on T2*-weighted images. The other condition that could have caused hyperperfusion and BOLD effect in our patient was the tonico-clonic seizure, but this could be ruled out given the characteristic pattern of occipital and anterior watershed hyperperfusion, together with the persistence of abnormal CBF and BOLD effect on D10 follow-up MRI. Our observation supports the pathophysiological theory of impaired cerebrovascular regulation during PRES, and provides a new MRI marker for the diagnosis of PRES. 


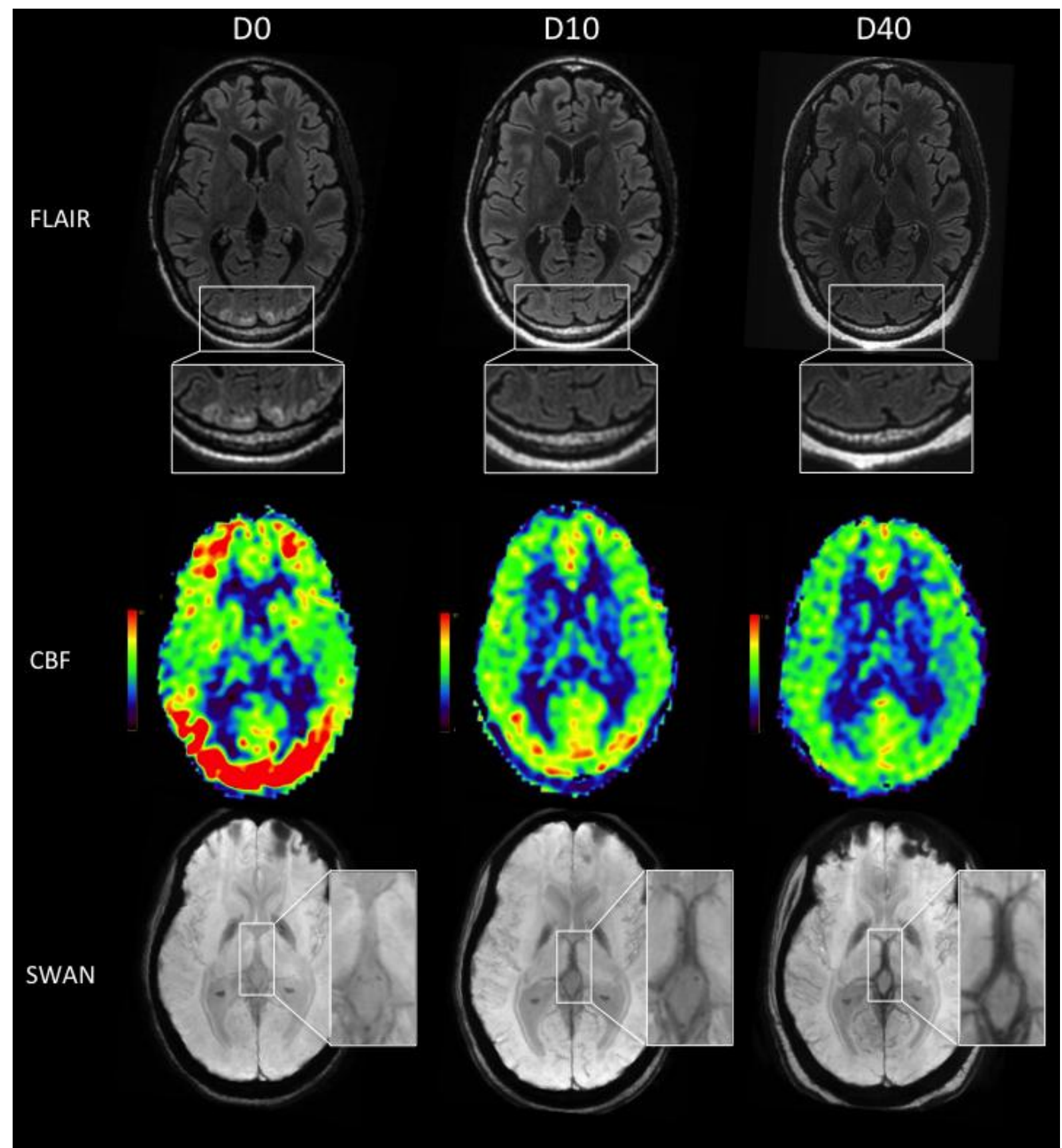

Figure 1. Initial and follow-up MRI at D0, D10 and D40 (Signa 3T HD23, GE Healthcare, Buc, France). Axial Fluid-Attenuated Inversion Recovery images (FLAIR); Arterial Spin Labelling Cerebral Blood Flow map (CBF); and axial T2 Star Weighted Angiography (SWAN) presented in $5 \mathrm{~mm}$-thick minimum intensity projection (minIP). Theprogressive decrease in signal of the venous system parallels the progressive normalization in 
cerebral perfusion, demonstrating a transient BOLD effect linked to cerebral hyperperfusion.

\section{REFERENCES}

[1] Bartynski WS. Posterior reversible encephalopathy syndrome, part 1: fundamental imaging and clinical features. AJNR Am J Neuroradiol 2008;29:1036-42. doi:10.3174/ajnr.A0928.

[2] Deibler AR, Pollock JM, Kraft RA, Tan H, Burdette JH, Maldjian JA. Arterial spinlabeling in routine clinical practice, part 3: hyperperfusion patterns. AJNR Am J Neuroradiol 2008;29:1428-35. doi:10.3174/ajnr.A1034.

[3] Buxton RB. Dynamic models of BOLD contrast. NeuroImage 2012;62:953-61. doi:10.1016/j.neuroimage.2012.01.012. 